\title{
STEM and EELS Investigation on Black Phosphorus at Atomic Resolution
}

\author{
Giuseppe Nicotra ${ }^{1}$, Antonio Massimiliano Mio ${ }^{1}$, Anna Cupolillo ${ }^{2}$, Jin $\mathrm{Hu}^{3}$, Jiang $\mathrm{Wei}^{3}$, Zhiqiang Mao ${ }^{3}$, \\ Ioannis.Deretzis ${ }^{1}$, Antonio Politano ${ }^{2}$, and Corrado Spinella ${ }^{1}$ \\ ${ }^{1}$ CNR-IMM, Strada VIII, 5, 95121 Catania, Italy \\ ${ }^{2}$ Dipartimento di Fisica, Università della Calabria, 87036 Cosenza, Rende, Italy \\ ${ }^{3}$ Department of Physics, Tulane University, New Orleans LA 70118 USA
}

Recently, black phosphorus has attracted a huge attention for the discovery of unusual properties with high potential for microelectronics [1-4]. Black phosphorus is the thermodynamically more stable phase of phosphorus, at ambient pressure and temperature. Black phosphorus is a narrow-gap semiconductor with orthorhombic structure. It is a layered material, with each layer forming a puckered surface due to $\mathrm{sp}_{3}$ hybridization.

The three bonds take up all three valence electrons of phosphorus, so unlike graphene, a monolayer black phosphorus (termed "phosphorene") is a semiconductor with a direct band gap of $1 \mathrm{eV}$ at the $\Gamma$ point of the first Brillouin zone. For few-layer phosphorene, interlayer interactions reduce the band gap for each layer added, and eventually reach $\sim 0.3 \mathrm{eV}$ for bulk black phosphorus. The direct gap also moves to the $\mathrm{Z}$ point as a consequence. The presence of an energy gap is crucial for the field-effect transistor (FET) application of two dimensional (2D) materials, while for example graphene does not have an energy gap. Moreover, the thickness-dependent direct band gap may lead to potential applications in optoelectronics, especially for THz applications.

Plasmons in black phosphorus have not been experimentally studied yet. Only a plasmon satellite at 20 $\mathrm{eV}$ has been identified in 2s and 2p core levels of P in X-ray photoemission spectroscopy (XPS) [5]. Moreover, STEM and EELS experimental end theoretical study are missing yet.

For the first time, the direct atomic structure and electronic structure of the black phosphorus were studied and the results presented in cross-section. All the STEM and atomic EELS measurements were performed at $60 \mathrm{kV}$ and $200 \mathrm{keV}$ by state-of-the-art aberration-corrected microscope installation at Beyond-Nano sub-Ångstom Lab, in Catania, Sicily, Italy. This consists of a probe corrected STEM microscope equipped with a C-FEG and a fully loaded GIF Quantum ER as EELS spectrometer. This particular installation is capable to deliver a probe size of $0.68 \AA$ at $200 \mathrm{kV}$, and $1.1 \AA$ at $60 \mathrm{kV}$. Low and core-loss EELS spectra were nearly simultaneously acquired using the DualEELS capability. Figures 1 a) shows the simulated side view of the structure of bulk black phosphorus. Figures 1b) shows the experimental direct atomic structure acquired in STEM HAADF mode at 200keV of beam acceleration voltage with an illumination angle of 33mrad and a collection angle of $20 \mathrm{mrad}$.

Figures 2 a) show the plasmon peaked at $20 \mathrm{eV}$ and Figures b) shows the $\mathrm{P} \mathrm{L}_{2.3}$ at $132 \mathrm{eV}$ and the $\mathrm{P} \mathrm{L}_{1}$ at $189 \mathrm{eV}$, both acquired with an illumination angle of 33mrad and a collection angle of 10mrad. Ab-initio simulation through FEFF of the EELS spectra will be also presented.

References

[1] Xia F, Wang H, Jia Y. Rediscovering black phosphorus as an anisotropic layered material for optoelectronics and electronics. Nat Commun. 2014;5:4458.

[2] Buscema M, Groenendijk DJ, Blanter SI, Steele GA, Van Der Zant HSJ, Castellanos-Gomez A. Fast 
and broadband photoresponse of few-layer black phosphorus field-effect transistors. Nano Lett. 2014;14(6):3347-52.

[3] Fei R, Yang L. Strain-engineering the anisotropic electrical conductance of few-layer black phosphorus. Nano Lett. 2014;14(5):2884-9.

[4] Han X, Morgan Stewart H, Shevlin SA, Catlow CRA, Guo ZX. Strain and orientation modulated bandgaps and effective masses of phosphorene nanoribbons. Nano Lett. 2014;14(8):4607-14.

[5] Asahina H, Morita A. Band structure and optical properties of black phosphorus. J Phys C. 1984;17(11):1839-52.

[6] This work was performed at Beyondnano CNR-IMM, which is supported by the Italian Ministry of Education and Research (MIUR) under project Beyond-Nano (PON a3_00363).
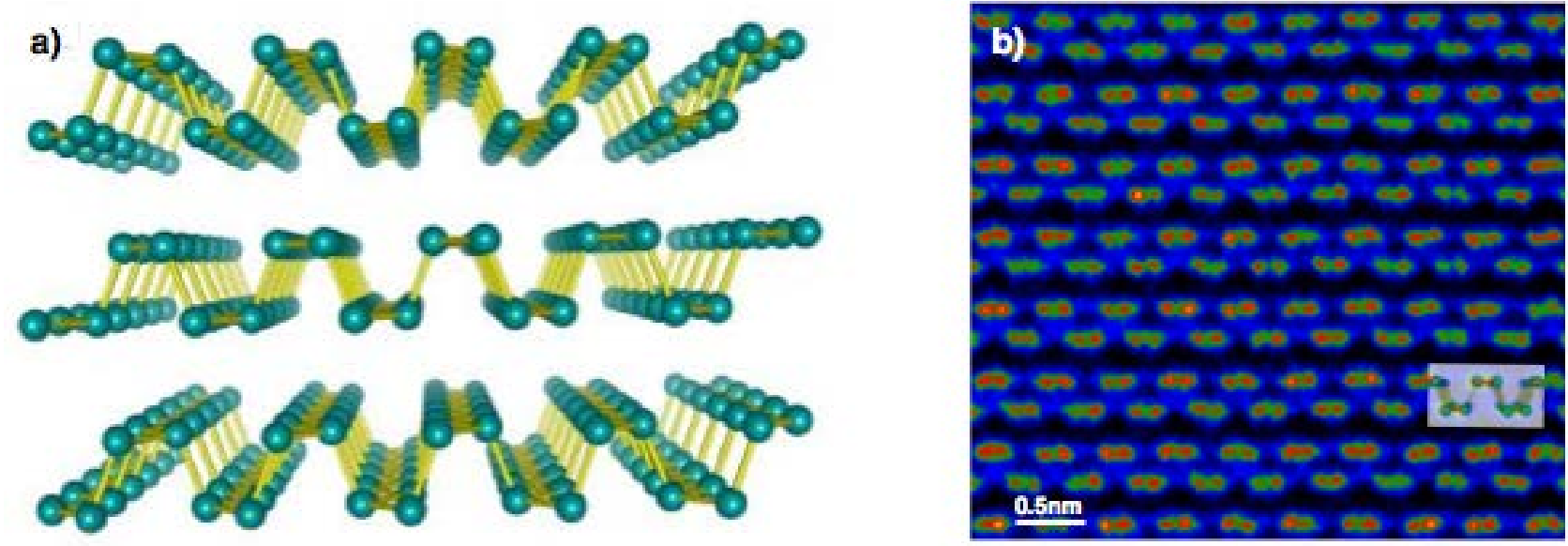

Figure 1. a) Side view of the structure of bulk black phosphorus. b) cross-sectional view (HAADF STEM image) of bulk black phosphorus clearly showing the armchair arrangement of the $\mathrm{P}$ atoms.
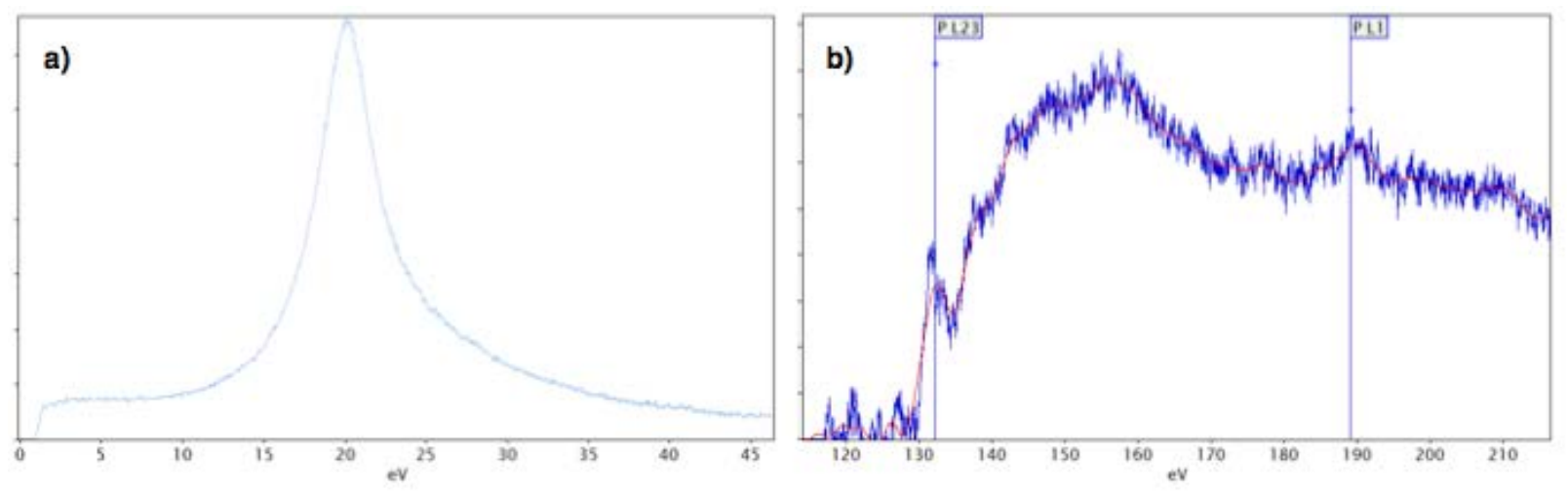

Figure 2. a) Plasmon and b)P L spectrum of black phosphorus 